\title{
PERSPECTIVAS DE RISCO E BENÉFICIOS DE UMA COMUNIDADE RURAL SOBRE O PROJETO DE INTEGRAÇÃO DE BACIAS DO RIO SÃO FRANSCISCO
}

\author{
Gleydson Kleyton Moura Nery ${ }^{1}$ \\ Janiele França Nery ${ }^{2}$ \\ Evaldo de Lira Azevêdo 3
}

\begin{abstract}
RESUMO
O estudo teve como objetivo avaliar as percepções de risco e benefício de uma comunidade rural sobre o projeto de integração das bacias hidrográficas do São Francisco na Paraíba. Para isto, testamos a hipótese de que as percepções são influenciadas pelas atividades ocupacionais e gênero dos participantes. As comunidades rurais do entorno do reservatório de Poções (Monteiro - PB), o qual é o receptor das águas do projeto de integração do rio São Francisco no estado, foram visitadas entre agosto e outubro de 2015 . Os moradores foram questionados sobre as possibilidades de riscos e benefícios do projeto para resolver o problema de água na região. As respostas foram agrupadas em categorias e foram calculados os índices de incidência de risco, de benefício e beneficência. A percepção de risco dos moradores teve baixa incidência, embora o gênero tenha influenciado a percepção de risco da comunidade, sendo os problemas sociais e ambientais, melhor percebidos pelas mulheres enquanto a maioria dos homens não conseguiu identificar riscos associados ao projeto. A disponibilidade de água é o benefício mais incidente, seguido das possibilidades de atividades ocupacionais. Para o índice de beneficência, a atividade ocupacional influenciou as percepções. Pescadores e aposentados foram associados à variável atividade ocupacional, enquanto domésticas e agricultores foram associados principalmente às variáveis disponibilidade e qualidade da água. Não há diferenças significativas relacionadas à questão de gênero para benefícios, embora a maioria das mulheres tenha apontado benefícios relacionados à disponibilidade de água.
\end{abstract}

Palavras-chave: Conhecimento tradicional. Gênero. Atividade ocupacional. Semiárido

\footnotetext{
1 Biólogo e Mestre em Ecologia e Conservação pela Universidade Estadual da Paraíba - UEPB, Pesquisador do Núcleo de Recursos Hídricos do Instituto Nacional do Semiárido - INSA. Autor para correspondência: gleydson.kleyton@gmail.com

${ }^{2}$ Bióloga e Doutora em Ciências Ambientais pela Universidade Estadual de Maringá - UEM. Pesquisadora do Núcleo de Recursos Hídricos do Instituto Nacional do Semiárido - INSA.

${ }^{3}$ Biólogo e Doutore em Etnobiologia e Conservação da Natureza pela Universidade Federal Rural do Pernambuco - UFRPE, Professor do Instituto Federal de Educação, Ciência e Tecnologia da Paraíba IFPB.
}

R. gest. sust. ambient., Florianópolis, v. 10, n. 2, p. 189-208, jun. 2021. 


\title{
RISK AND BENEFITS PERSPECTIVES OF A RURAL COMMUNITY ABOUT SÃO FRANSCISCO RIVER BASIN INTEGRATION PROJECT
}

\begin{abstract}
This study aimed to evaluate the risk and benefit perceptions of local villagers about São Francisco river basins integration project. We tested the hypothesis that the perceptions are influenced by occupation activities and gender of participants. Local villages around Poções reservoir (Monteiro- PB), first receiver of integrated waters of São Francisco project, were visited between August and October 2015. Villagers were asked about possibilities of risks and benefits of project to solve waters problem in region, using a questionnaire. The responses were grouped into categories and the incidence of risk, benefit and beneficence indices were calculated. The risk perception of the villagers had a low incidence, although, the gender influenced people's perception of risk. Social and environmental problems are important to women and most of the man no identify risks to project. Water disponibility is the most incident benefit, followed by possibilities of occupational activity. To benefit incidence, occupational activity influenced perceptions. Fishermen and retirees were associated with the variable occupational activity, while housekeepers and farmers were mostly associated with the variables availability and water quality. No significant differences related to the gender issue for benefits, although most women have pointed out benefits related to water availability.
\end{abstract}

Keywords: Traditional knowledge. Gender. Occupational activity. Semiarid

\section{INTRODUÇÃO}

O cenário hídrico para comunidades rurais em regiões semiáridas se caracteriza por baixo regime pluviométrico, escassez hídrica constante e depósitos de aquíferos raros e rasos (MOURA et al., 2007). No entanto a problemática da água nesta região, relaciona-se não só as condições ambientais como também a condução das políticas públicas, como a universalização do acesso e uso da água onde a disponibilidade de água de qualidade e em quantidade é restrito (REBOUÇAS, 2001; ALEIXO et al., 2015).

Neste contexto, o Projeto de Integração de Bacia Rio São Francisco com as Bacias Hidrográficas do Nordeste Setentrional (PISF) - popularmente conhecido como Transposição das águas do rio São Francisco, surge como uma proposta política que visa descentralizar, democratizar e assegurar a oferta de água a cerca de 12 milhões de habitantes do semiárido brasileiro (CONSÓRCIO ECOPLAN-SKILL, 2011), em consonância com a agenda pós-2015 que tem como objetivo eliminar progressivamente as desigualdades do acesso a água até 2030 (WHO, 2014).

R. gest. sust. ambient., Florianópolis, v. 10, n. 2, p. 189-208, jun. 2021. 
Estando o rio São Francisco presente já em cinco unidades federativas (MG, $\mathrm{BA}, \mathrm{PE}, \mathrm{AL}$ e SE) e abastecendo cerca de 14 milhões de pessoas em um território de $2.700 \mathrm{Km}^{2}$, a obra do PISF teve como objetivo expandir a sua área de contribuição através da construção de dois eixos de ligação, o eixo Norte com extensão 402 Km² e o eixo Leste com extensão de $220 \mathrm{Km}^{2}$, em que as águas do rio São Francisco são captadas no estado de Pernambuco, entre as barragens de Sobradinho e Itaparica, e direcionadas ao abastecimento das múltiplas bacias hidrográficas das unidades federativas do Ceará, Paraíba, Pernambuco e Rio Grande do Norte (BRASIL, 2004; MEIRON, 2009, TALLMAN, BENEDICTO, 2018).).

O fato da rede hidrográfica do Nordeste Setentrional ser predominantemente de rios intermitentes, ou seja, secam durante os meses em que não há precipitação, representam uma maior vulnerabilidade as secas no Brasil e sendo uma região que possui apenas políticas públicas de mitigação e combate à seca mas, não de promover a independência socioeconômicas (ANA, 2017; DANTAS; 2020), há inúmeros benefício a serem trazidos pela execução do PISF, podendo destacar o atendimento as demandas hídricas para as áreas urbanas, distritos industriais e perímetros de irrigação como também aos usos múltiplos nas zonas rurais ao longo dos canais, rios perenizados e reservatórios (CASTRO, 2011).

Apesar dos impactos positivos destacado pela implantação PISF existem consequências a serem consideradas, desde os (i) impactos socioeconômicos, como a introdução de tensões e riscos sociais durante a fase de obra, risco de interferência com o patrimônio cultural, desapropriação das comunidades rurais e a modificações da dinâmica de uso e pagamento pelos recursos hídricos; aos (ii) impactos ambientais, como modificações na composição das comunidade biológicos, riscos de redução da biodiversidade, introdução de espécies exóticas, modificação do regime fluvial, mudanças na dinâmica de sedimentação, trofia e qualidade de água dos reservatório e a perda e fragmentação de área vegetada para construção (BRASIL, 2004; SILVA et al., 2020; BARBOSA et al., 2021).

Apesar das medidas aplicadas pelo Governo, justificadas pelo caráter emergencial da região semiárida, torna-se cada vez mais claro o fato de muitas das ações, ocorrem sem uma discussão prévia, clara e inclusiva as comunidades sujeitas as pressões socioeconômicas e ambientais da região (BURNEY et al., 2014). Deste

R. gest. sust. ambient., Florianópolis, v. 10, n. 2, p. 189-208, jun. 2021. 
modo, a avaliação da problemática em relação a disponibilidade de água ultrapassa o balanço entre a oferta e a demanda, sendo necessário integrar as relações geoambientais e socioculturais, com objetivo reduzir a vulnerabilidade social e garantir a qualidade e desenvolvimento socioeconômico e ambiental do capital ecológico (REBOUÇAS, 1997; SILVA et al., 2020)

As relações ente as comunidades e os recursos naturais são cada vez mais recorrentes, e apesar desse intricamento o processo de participação social e reconhecimento por parte do poder público como uma alternativa de contribuição com foco na sustentabilidade hídrica, ocorreu apenas recentemente, uma vez que, integrando populações tradicionais e locais, respeitando e valorizando seu conhecimentos e vivências locais, há possibilidade de geração de impactos duradouros na independência quanto ao uso e gestão dos recursos hídricos (SANTANA, 2013; MACHADO, LA ROVERE, 2018).

Sendo assim, a percepção mostra-se como uma importante ferramenta na compreensão e avaliação das interações socioambientais, uma vez que, envolve a exposição do indivíduo/grupo a estímulos assim como, sua aproximação com ambiente (ALBUQUEERQUE et al., 2014; ESPINOSA-GARCI et al., 2015). Tais exposições em que o indivíduo/grupo é exposto a condição de perigo ou risco passa a ser conhecido como percepção de risco, sendo um elemento natural do contexto cultural dos indivíduos, podendo ser, influenciado por inúmeros fatores como crenças, emoções, familiaridade e consequências de eventos/condições, medo e grau de consciência do risco (ALBUQUERQUE et al., 2014; RAMBONILAZA et al., 2016)

A avaliação do risco baseada na percepção não é apenas uma questão de percepção sensorial, mas sim embasada nas atitudes e expectativas do indivíduo, que através da medição de atitude em escala psicológica, com uso de questionários e escalas possibilita o mapeamento participativo de risco (ALBUQUERQUE et al., 2014). O uso da avaliação de risco tem sido muito utilizado como base para decisões regulamentadoras na avaliação ambiental com foco na viabilidade social, contudo, a sua avaliação quantitativa mostra-se complexa e permanece sujeita a debate, justamente devido aos elementos psicológicos, socioculturais e ambientais a serem considerados (BURGUER et al., 1999, RAMBONILAZA et al., 2016).

Podendo assim destacar que, em grande projetos, a exemplo da PISF, mudanças são esperadas tanto no modo de vida como em alterações territoriais, mas R. gest. sust. ambient., Florianópolis, v. 10, n. 2, p. 189-208, jun. 2021. 
que também, contrastam aos benefícios propiciados, sendo assim necessário detectar as condições em que cada indivíduo está sujeito podendo diagnosticar seus conflitos de interesse e respectivamente a sua percepção em relação a realidade em que se encontra (SILVA et al., 2020). Com isso, este trabalho tem como objetivo avaliar as percepções de risco e de benefício da comunidade rural, do município de Monteiro, em relação ao projeto de integração de bacias com Rio São Francisco na Paraíba. Para isto, testamos a hipótese de que a percepção dos indivíduos apresente diferenças considerando gênero e atividade ocupacional.

\section{MATERIAL E MÉTODOS}

\section{1 Área Estudo}

Com objetivo de compreender previamente a percepção social em relação ao Projeto de Integração de Bacia Rio São Francisco com as Bacias Hidrográficas do Nordeste Setentrional (PISF), o estudo foi conduzido com comunidades rurais do entorno do reservatório de Poções, localizado no município de Monteiro-PB ( $7^{\circ} 53^{\prime} 33^{\prime \prime} \mathrm{S} 37^{\circ} 0^{\prime} 32$ "O), o qual é o primeiro reservatório do estado a receber as águas do rio São Francisco por meio do eixo leste.

O eixo leste possui um extensão territorial de $220 \mathrm{Km}^{2}$ em que direciona as água do São Francisco ao estado da Paraíba, beneficiando 169 município e uma população estimada em 31.500 pessoas até 2026 distribuídas em comunidades nos municípios de Floresta, Custódia, Betânia e Sertânia (estado de Pernambuco) e oito comunidades no município de Monteiro, no estado da Paraíba , sendo o primeiro sistema a entrar em funcionamento, em março de 2017, abastecendo a bacia do rio Paraíba através de cinco reservatórios: São José I, Poções, Camalaú, Boqueirão e Acauã (BRASIL, 2004; CONSÓRCIO ECOPLAN-SKILL, 2011; BARBOSA et al., 2021).

R. gest. sust. ambient., Florianópolis, v. 10, n. 2, p. 189-208, jun. 2021. 
Figura 1. Localização geográfica da área de estudo - Açude de Poções e sua comunidade no entorno, Monteiro, PB.
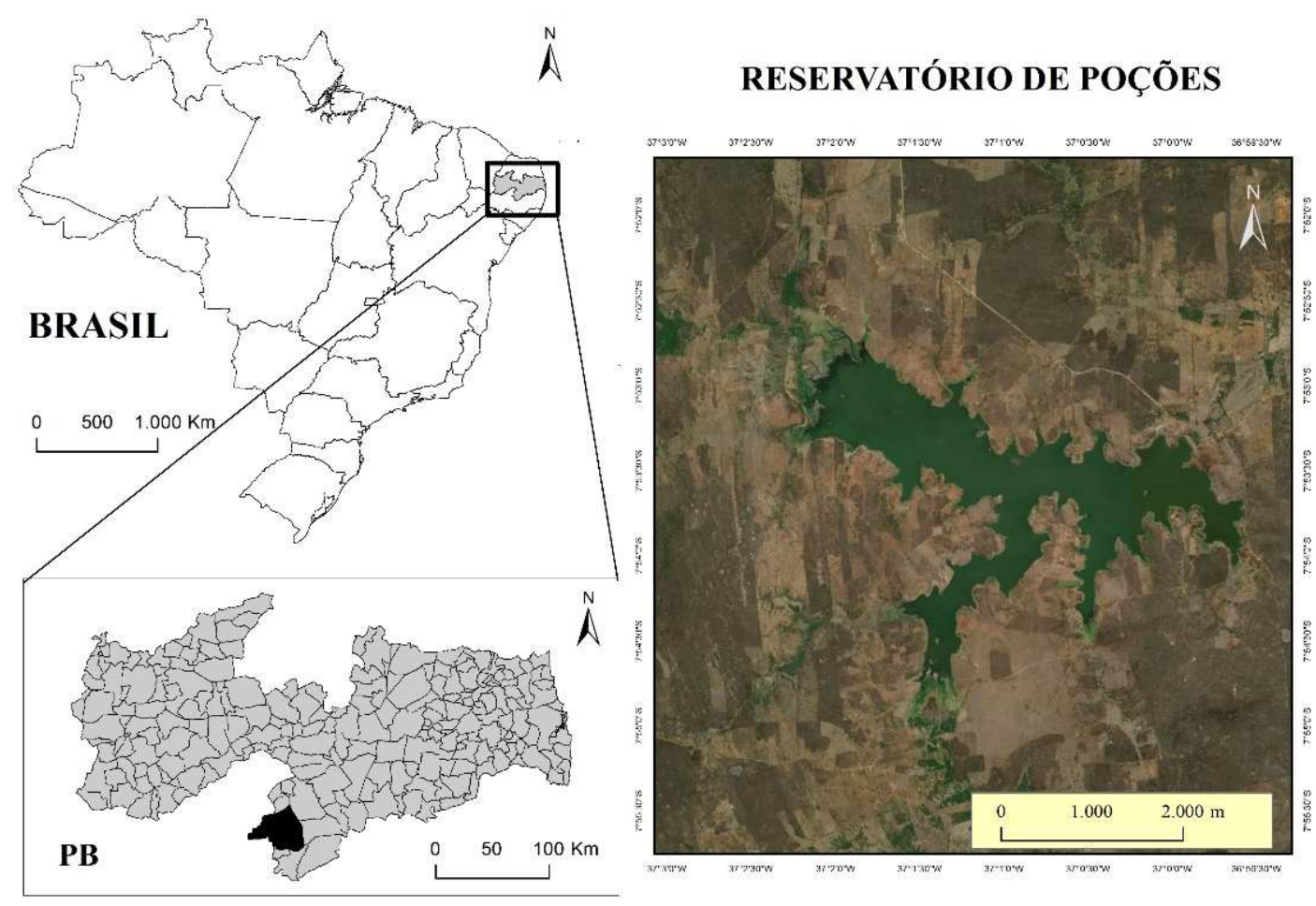

Para a área de estudo, a qual caracteriza-se como região semiárida, a precipitação é bem definida apresentando uma estação seca com recorrentes eventos de escassez de água, e uma estação chuvosas, com uma distribuição desigual das precipitações, com chuvas intermitentes que somam entre 280 e $800 \mathrm{~mm} /$ ano (PEREIRA-JUNIOR, 2007; SILVA et al., 2018). A classificação climática (Köppen) para esta região é Bsh e tipo tropical semiárido (MENDONÇA, OLIVEIRA, 2007; ALVARES et al., 2013).

Entre os anos de 2012 e 2016 foi registrado uma das mais severas secas da história da região, onde o principal efeito foi a crise de abastecimento público, onde para reservatório de Poções (Monteiro - PB) que possui a capacidade de abastecimento de $29.860 .000 \mathrm{~m}^{3}$ para o suprimento de água de 40 cidades do estados, durante o ano de 2015 chegou a apresentar menos de $1 \%$ da sua capacidade (105.770 $\mathrm{m}^{3}$ ), resultando com perdas de produção e consequências socioeconômicas importantes, visto que a principal fonte de renda da região se concentra na agricultura

R. gest. sust. ambient., Florianópolis, v. 10, n. 2, p. 189-208, jun. 2021. 
de subsistência, tornando a necessidade de transferência das água do rio São Francisco emergencial (AESA, 2021; DANTAS et al., 2020).

\subsection{Plano Amostral e Coleta de Dados}

Para a coleta de dados, as informações foram obtidas via moradores locais por meio de entrevistas semiestruturadas com uso de questionário contendo questões abertas e fechadas sobre o Projeto de Integração de Bacia Rio São Francisco com as Bacias Hidrográficas do Nordeste Setentrional (PISF) e os possíveis riscos e/ou benefícios da obra para solução hídrica da região. Para validação do formulário foi realizada uma aplicação com uma subamostra do universo amostral na primeira expedição ao campo da pesquisa, para adequação de tempo de aplicação e a criação do "rapport", elo de confiança e empatia entre o entrevistador e o entrevistado, minimizando assim o erro de interpretação e informações incorretas.

Foram visitadas todas as residências situadas até $150 \mathrm{~m}$ do reservatório entre os meses de agosto e outubro de 2015. Antes de cada entrevista os objetivos da pesquisa foram explicitados da mesma forma que foi obtida a permissão através do Termo Livre Esclarecido (TCLE) para registrar as respostas por meio de áudio e imagens, segundo a Resolução 466/20124.

Em cada residência foi entrevistado pelo menos um indivíduo por um período máximo de $45 \mathrm{~min}$, preferencialmente chefes de família (homens ou mulheres) totalizando o universo amostral de 40 entrevistados. Destaca-se que respostas ao questionário podem ter sido influenciadas em decorrência dos últimos 3 anos de seca até o memento da realização da pesquisa na região Semiárida.

\subsection{Análise de Dados}

\subsection{1 Índice de incidências de risco, benefício e beneficência}

A análise da percepção de risco foi utilizada para identificar, classificar e ordenar os fatores de risco percebidos pela comunidade, seguindo a equação:

\footnotetext{
${ }^{4} \mathrm{~A}$ aprovação de ética para o projeto de pesquisa foi concedida pelo Comitê de Ética da Universidade Estadual da Paraíba - UEPB, Brasil ( $n^{\underline{a}}$ de protocolo de aprovação: 44582415.6.0000.5187).
}

R. gest. sust. ambient., Florianópolis, v. 10, n. 2, p. 189-208, jun. 2021. 


$$
\boldsymbol{l} \boldsymbol{j}=(n r / n \boldsymbol{j})
$$

onde: lj = Índice de Incidência de Risco, $\mathrm{nr}$ = número de vezes que um dado risco foi citado e nj = número total de entrevistados. Os valores de lj podem variar entre 0 e 1 , com os valores mais altos correspondendo a riscos percebidos mais elevados (ALBUQUERQUE et al., 2014).

Para a análise complementar da percepção dos entrevistados foi proposta 0 desenvolvimento de índices que calcule a percepção de benéficos com base em Albuquerque et al., (2014), no qual tais índices permitem a identificação, classificação e ordenamento dos fatores de benefícios percebidos pela comunidade em relação a PISF. Para isto, seguiu-se as seguintes etapas: (i) identificação dos fatores de benefícios mencionados por cada entrevistado; (ii) organização dos benefícios citados em ordem de importância citada pelo entrevistado e o (iii) cálculo da incidência de benefício $\left(I_{B}\right)$ e do índice de beneficência $(B)$, seguindo as equações:

$$
I_{B}=(n b / n j)
$$

onde $n b$ é o número de vezes que o benefício foi citado e nj o número de todos os entrevistados. Deste modo, os valores de $I_{B}$ podem variar entre 0 e 1, assim os valores próximos de 0 tem uma menor frequência de citação e aqueles mais próximos de 1 tem maior frequência de citação. Este índice implica na proporção de entrevistados que identificam determinado fator de benefício, mas sem considerar necessariamente o grau de benefício que o mesmo causa.

$$
B \boldsymbol{j}=1+(\mathrm{b}-1) /(\mathrm{n}-1)
$$

Esse índice é baseado no número e ordenamento dos fatores de benefícios citados por cada entrevistado, variando entre 1 e 2 , sendo os valores mais próximos de 1 considerados mais benéficos onde $b$ é o ordenamento baseado na ordem indicada pelo respondente e $n$ é o número de fatores de benefício que foram mencionados pelo respondente. Permitindo identificar o grau de benefício causado pelo fator citado.

Portanto, a média dos valores de $B$ é calculada para o subconjunto de pessoas que identificaram o benefício. Esse índice é baseado no número e ordenamento dos fatores de benefício citados por cada entrevistado, variando entre 1 e 2, sendo os valores mais próximos de 1 considerados mais benéficos. Ao final dos cálculos os benefícios foram representados graficamente.

R. gest. sust. ambient., Florianópolis, v. 10, n. 2, p. 189-208, jun. 2021. 


\subsubsection{Análise estatísticas}

Uma Análise de Componentes Principais (PCA) foi utilizada com o objetivo de melhor compreender a natureza da relação entre as variáveis revelando a estrutura interna dos dados, de forma simplificar o entendimento da variabilidade das medidas para a percepção de riscos e benefícios da integração do rio São Francisco entre o gênero e as atividades ocupacionais. Todas as análises foram realizadas no software PRIMER 6 add Permanova ${ }^{\text {TM }}$ (CLARKE, GORLEY, 2006).

\section{RESULTADOS E DISCUSSÃO}

\subsection{Caracterização socioeconômica}

A maioria dos indivíduos entrevistados são do sexo masculino $(60 \%, n=24)$, enquanto os indivíduos do sexo feminino representaram $40 \%(n=16)$ dos entrevistados. A idade variou de 24 a 87 anos $(49,1 \pm 15,45)$. Dos entrevistados 22 , $5 \%(n=9)$ são analfabetos, $45 \%(n=18)$ não concluíram o ensino fundamental, $20 \%$ $(\mathrm{n}=8)$ não concluíram o ensino médio, $7,5 \%(\mathrm{n}=3)$ concluíram o ensino médio, 2,5\% $(n=1)$ possuíam ensino técnico e $2,5 \%(n=1)$ ensino superior incompleto. A principal fonte de renda da comunidade foi a agricultura e pecuária $(47,5 \%, n=19)$, aposentados $25 \%(n=10)$, pescadores, $12,5 \%(n=5)$, cuidadores domiciliares $12,5 \%$ $(n=5)$ e trabalhadores autônomos $2,5 \%(n=1)$, com renda média de $R \$ 735,71$. $O$ tempo de residência nos locais amostrados variou de 1 a 87 anos $(28,43 \pm 20,75)$.

\subsection{Percepção dos riscos e benefícios}

A identificação dos riscos é essencial para o desenvolvimento e foco de projetos, especialmente aqueles que visam o favorecimento da qualidade de vida de comunidades em vulnerabilidade, de forma que, sem compreender as percepções locais é improvável que os projetos obtenham sucesso em seu desenvolvimento (QUINN et al., 2003).

Apesar de ser considerada uma das obras mais custosas do governo federal, o PISF sempre foi representado como uma obra de impacto em dimensões sociais, econômicos, ambientas e culturais, principalmente no que se refere ao sertanejo da região semiárida, a qual contribui para um severa mudança no que se refere a disputas

R. gest. sust. ambient., Florianópolis, v. 10, n. 2, p. 189-208, jun. 2021. 
por posse de água, expansão agrária e da pecuária (CARVALHO; MENANDRO, 2018).

Portanto, a análise da incidência de risco identificou como categorias principais (i) não há risco, (ii) desalojamento e (iii) controle e pagamento pelo uso do recurso. Apesar da ampla discussão na mídia acerca dos malefícios e benefícios do projeto de integração do São Francisco, em especial durante as campanhas políticas presidenciais ocorridas entre 1998 e 2012 (HENKE, 2013), há uma subestimação dos riscos desse projeto pelos entrevistados. Notadamente os riscos com maiores fatores de incidência relacionam-se a aspectos socioeconômicos, sendo os danos ambientais pouco percebidos (Tabela 1).

Tabela 1. Valores da incidência de risco (IR) percebidos pelos entrevistados da comunidade do entorno do reservatório de Poções em Monteiro - PB.

\begin{tabular}{c|c}
\hline FATORES & INCIDÊNCIA DE RISCO (IR) \\
\hline Não há risco & 0,55 \\
\hline Desalojamento & 0,20 \\
\hline Controle \& Pagamento pelo recurso & 0,15 \\
\hline Secar o Rio São Francisco & 0,10 \\
\hline Perda da qualidade da água & 0,0025 \\
\hline
\end{tabular}

Fonte: Dados da Pesquisa (2015)

Em geral, a gravidade dos riscos percebidos aumenta de acordo com sua incidência, uma vez que a percepção individual está atribuída a condições de influências, que se comportam como filtros na identificação e difusão da informação na comunidade, reafirmando-se como risco ou não na construção sociocultural (HANNIGAN, 2009; GUEDES et al., 2015).

A predominância de respostas relativas à ausência de riscos, devem estar associadas a ideia de segurança hídrica diante das secas da região semiárida, a qual encontra-se alicerçada na formação da proposta do PISF e tem-se intensificado

R. gest. sust. ambient., Florianópolis, v. 10, n. 2, p. 189-208, jun. 2021. 
durante o período estudado. Tendo, as secas que acometem o Nordeste se tornado cada vez mais severas (1877-1879, 1998-1999, 2012-2017) promovendo quadros de ameaça de colapso no abastecimento hídrico através do colapso de açudes com volumes zerados, abastecimento por fontes alternativas (ex. carros-pipa), irrigação proibida e perdas críticas para agropecuária tornando-se manchete recorrente em toda a mídia regional e nacional e servindo como um dos pressores do PISF (MARENGO et al., 2016, CARVALHO; MENANDRO, 2018).

As secas identificadas no século XXI têm sido caracterizadas por temperaturas mais altas, duração mais prolongada e maior extensão espacial, sendo diretamente influenciadas pelas demandas por água (CRAUSBAY e RAMIREZ, 2017). Deste modo, a previsão de disponibilidade de água implica em mudanças no significado das percepções, as quais podem mudar rapidamente (ASHLEY E HUSSEIN, 2000).

A análise de componentes principais (PCA), baseada na percepção de risco dos entrevistados, explicou $76,2 \%$ da variabilidade dos dados nos dois primeiros eixos, sendo não há risco, controle e pagamento pelo recurso e desalojamento as variáveis mais correlatas aos eixos. A segregação dos casos foi independente da ocupação profissional, no entanto, observa-se a formação de grupos baseado no gênero, sendo as respostas dos homens predominantemente associadas a não há risco e controle e pagamento pelo recurso, enquanto as das mulheres foram mais diversas (Figura 2).

Figura 2. Análise de componentes principais (ACP) correlacionando os principais riscos com as atividades ocupacionais dos entrevistados da comunidade do entorno do reservatório de Poções em Monteiro - PB (H- homens entrevistados; M- mulheres entrevistadas).

R. gest. sust. ambient., Florianópolis, v. 10, n. 2, p. 189-208, jun. 2021. 


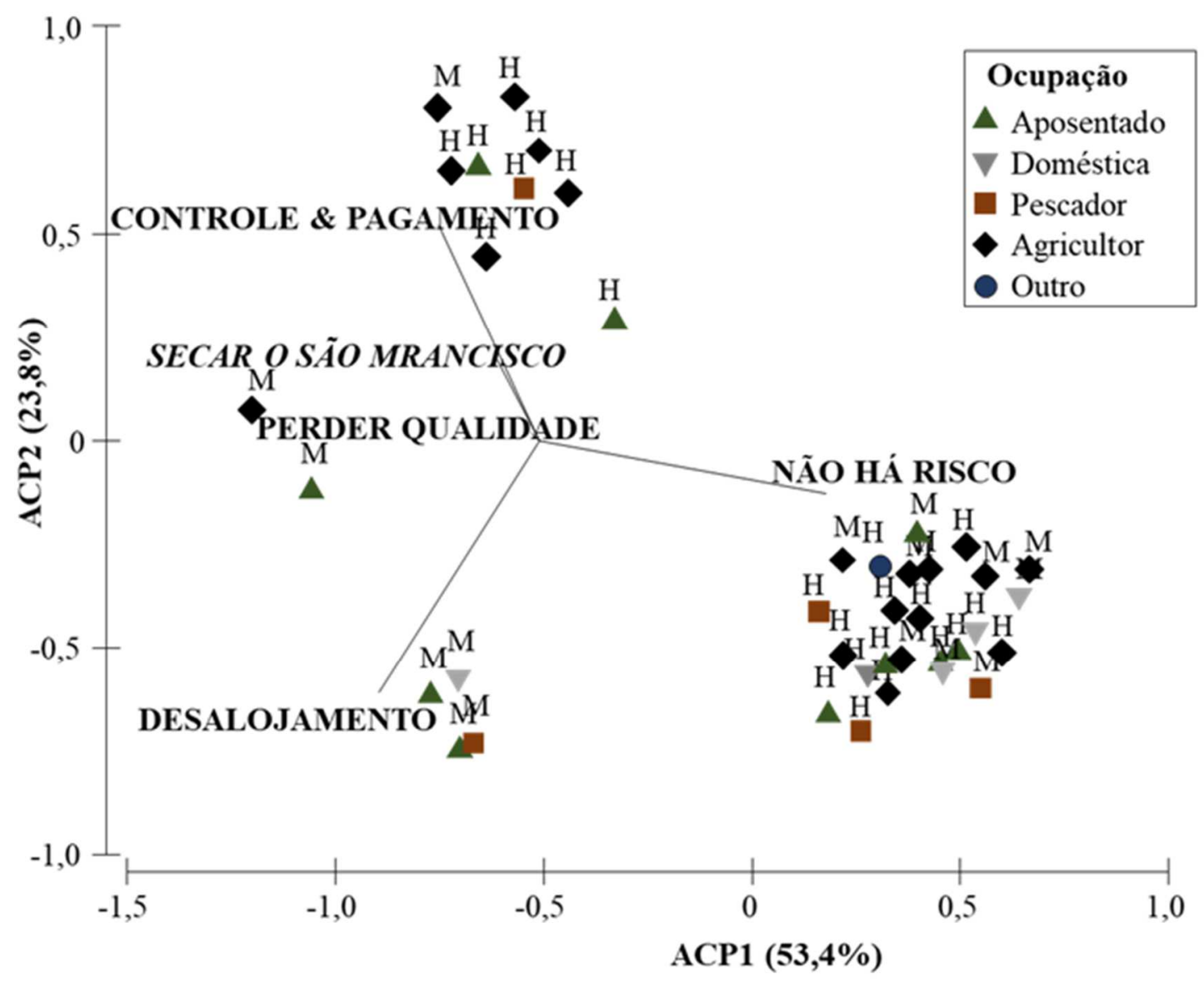

Fonte: Dados da Pesquisa (2015)

Quando nos referimos a percepção de risco em relação aos recursos hídricos temos como principal resposta modificações no padrão de uso da água, podendo ser associadas a fatores socioeconômicos, como gênero, idade, atividades ocupacionais entre outros (ARTELL et al., 2013; AFROZ et al., 2015). A perspectiva tradicionalista que configura o homem como provedor da casa e a mulher como dona da casa e cuidadora (NARAYAN et al., 2000), justifica a preocupação maior dos homens na prerrogativa de gestão dos custos e pagamentos pela água a ser disponibilizado e máscara o papel gestor da mulher, que tende a ser responsável por diversas esferas dentro da família e na comunidade em geral (NARAYAN et al., 2000; GALLO \& NAVARRO, 2018).

Para o semiárido, a mulher apresenta uma relação ainda maior com os recursos hídricos, representada em suas práticas tanto na busca, como na utilização e gerenciamento da água em uma realidade em que há desafios em sua qualidade e

R. gest. sust. ambient., Florianópolis, v. 10, n. 2, p. 189-208, jun. 2021. 
quantidade, tanto para o abastecimento como para os diversos usos (MELO, 2006), possibilitando-a de tornar-se mais perceptiva a questões sociais e ambientais.

Enquanto que, o homem como detentor das atividades ocupacionais na comunidade, restringe-se a perceber riscos associados a ocupação profissional que apresenta determinada influência sobre a capacidade de perceber modificações no contexto ambiental ao qual estão inseridas (JONES \& DUNLAP, 1992; SHI e HE, 2012)., ainda mais relativo as comunidades estudadas que apresentam predominantemente suas atividades ocupacionais relacionadas diretamente aos recursos hídricos (ex. pesca, agricultura e pecuária).

Enquanto, para os riscos relativos ao controle de acesso a água, desalojamento tornam-se compreensíveis a ambos os gêneros visto que, comunidades ribeirinhas tem acesso livre ao recurso dando maior flexibilidade nos usos da água, que em geral são utilizadas para a realização de serviços domésticos, a produção agrícola e até mesmo para o consumo humano.

Grandes obras de engenharia situam-se no campo da política onde se evidenciam conflitos de interesses envolvendo a apropriação privada dos recursos naturais de uso comum, tão como a expropriação dos territórios ocupados por povos e comunidades tradicionais e ribeirinhas (SILVA et al., 2020). Sendo, estes riscos causadores da necessidade de um reordenamento do ciclo social causando transtornos e assumindo todo um custo social e ambiental (ZHOURI; LASCHEFSKI, 2014).

Para os benefícios, a análise da variação representada (Figura 3), identifica a disponibilidade de água como fator que apresenta maior Índice de beneficência e Incidência do benefício, sendo, portanto, o fator mais importante para comunidade seguido da possibilidade de diversificação nas atividades ocupacionais/profissionais.

FIGURA 3. Mapa de benefícios percebidos pelos entrevistados através da correlação entre o Índice de beneficência $(B)$ e a Incidência do benefício $\left(I_{B}\right)$.

R. gest. sust. ambient., Florianópolis, v. 10, n. 2, p. 189-208, jun. 2021. 


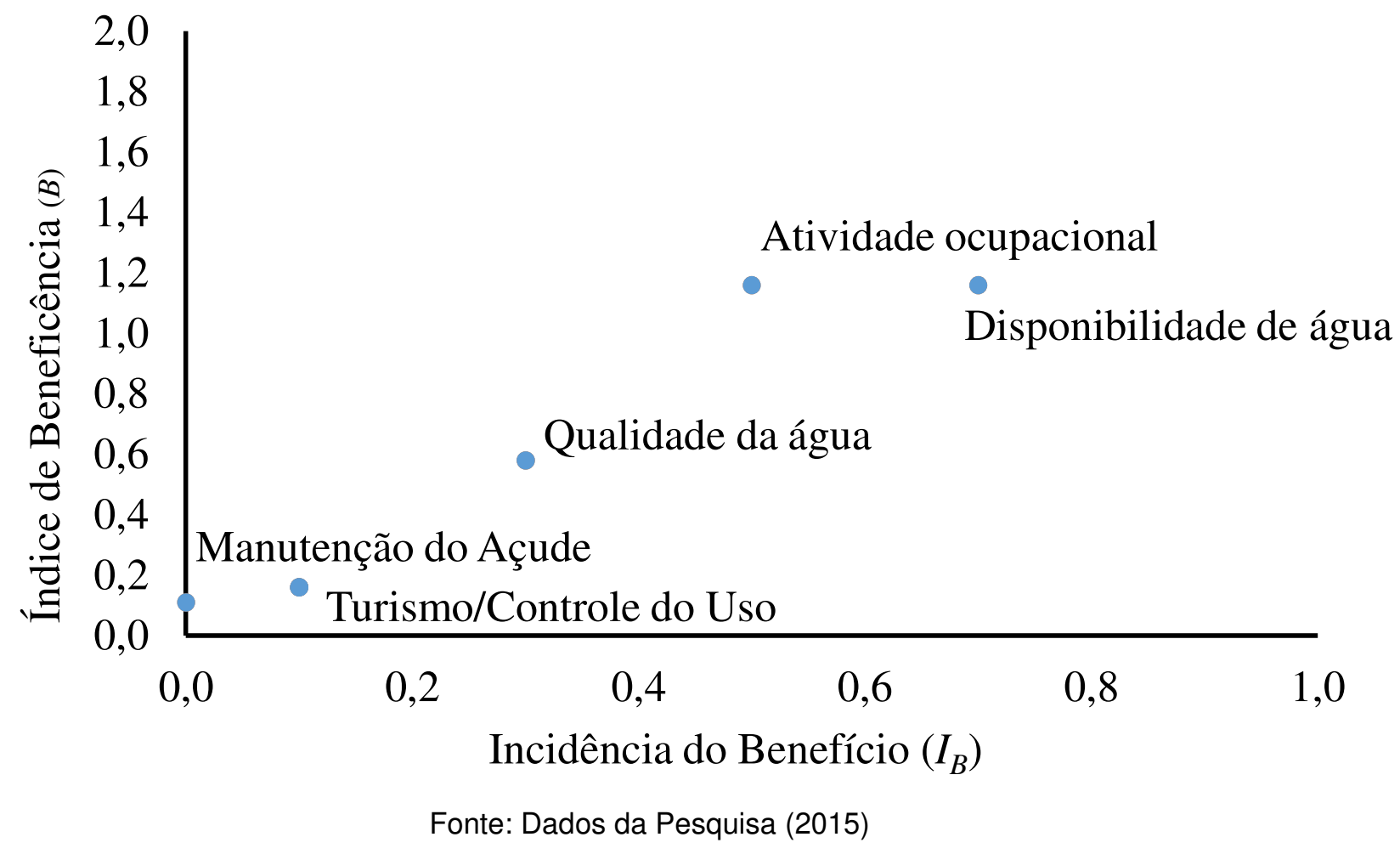

A percepção de benefícios pôde ser constatada, possivelmente em função do cenário hídrico desfavorável, onde a possibilidade de diversificação das atividades de subsistência é considerada para reduzir o risco e superar a instabilidade climática. $O$ estado de escassez hídrica apresenta reflexos na condição psicológica através dos sentimentos de insegurança e impotência, promovendo a necessidade de adaptação frente à redução de recursos até consequências a longo prazo, tendo como principal impacto prejuízos agrícolas e mudanças na rotina diária pelo racionamento de água e restrições na dieta alimentar (FAVERO E SARRIERA, 2014).

Prova disto, foi o período de seca mais recente em que abrangeu os anos de 2012 a 2017, sendo considerado um dos mais severos da história da região, tendo induzido uma série de consequências como a redução da do volume e qualidade hídrica - promovendo o colapso hídrico e comprometendo a produção agrícola e pecuária da região, em aproximadamente $U \$ 450$ milhões (DANTAS et al., 2020).

Considerando as variáveis de benefícios apontadas pelos entrevistados, a PCA explicou $75 \%$ da variabilidade dos dados nos dois primeiros eixos - disponibilidade de água, ocupação profissional e qualidade de água as variáveis mais correlatas aos eixos. A segregação dos casos apresentou formação de grupos associados a

R. gest. sust. ambient., Florianópolis, v. 10, n. 2, p. 189-208, jun. 2021. 
atividade profissional, de modo que pescadores e aposentados foram associados a variável atividade ocupacional/profissional, enquanto domésticas e agricultores estiveram majoritariamente associados as variáveis disponibilidade e qualidade de água (Figura 4). Ao contrário do observado para os fatores de risco, não foram evidenciadas diferenças significativas relacionadas a questão de gênero para os benefícios, ainda que, a maior parte das mulheres tenham apontado benefícios relacionados a disponibilidade de água.

Figura 4. Gráfico da análise de componentes principais (ACP) correlacionando os principais benefícios com as atividades ocupacionais $(\mathrm{H}$ - homens entrevistados; $\mathrm{M}$ mulheres entrevistadas).

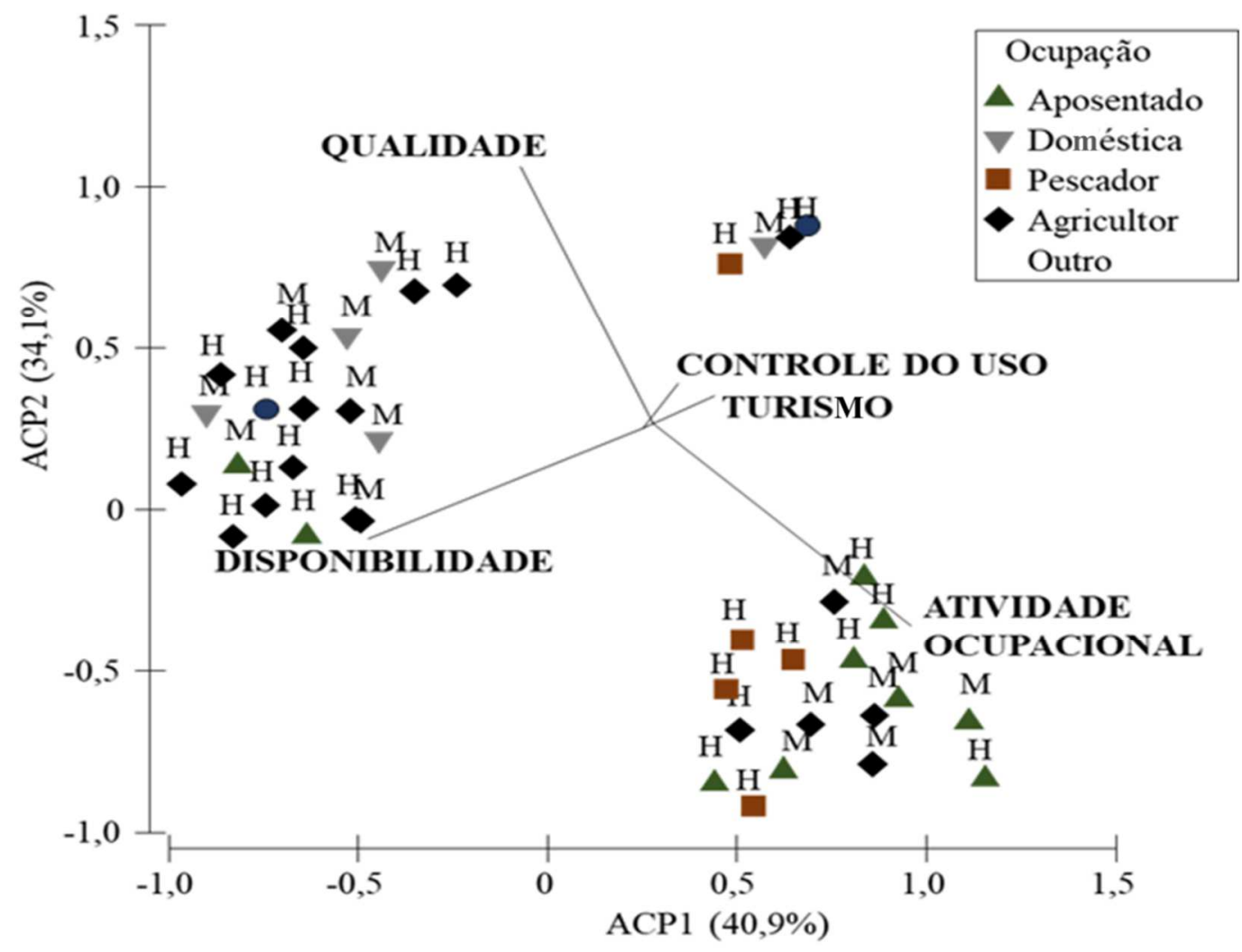

Fonte: Dados da Pesquisa (2015)

O acesso a água, de qualidade, tem se apresentado como um dos principais problemas para comunidades de regiões semiáridas, em particular para mulheres, que desempenham, primariamente, o papel de gestora das atividades domésticas diárias e muitas vezes auxílio nas atividades ocupacionais (QUINN et al., 2003), similiar ao observado neste estudo. Cabe considerar que o fenômeno das secas recorrentes na R. gest. sust. ambient., Florianópolis, v. 10, n. 2, p. 189-208, jun. 2021. 
região dificultam a recuperação das famílias, dificultando a construção de estratégias, principalmente no que se refere a reservas financeiras, sendo este o fator que contribui para uma maior vulnerabilidade a condição e percepção da disponibilidade e 0 controle e uso sobre o ambiente (FAVERO E SARRIERA, 2014, SILVA et al., 2020). Portanto, com objetivo de perenização do rio Paraíba no PISF como um forma de disponibilização contínua de "universalização" e acesso a água, apesar de quaisquer impactos subestimados nos relatório de impactos obra, os benefícios propostos superestimam aos impactos decorrentes do empreendimento de forma a não inviabilizar o projeto (CASTRO, 2011).

No que se refere as populações rurais, as secas e outros eventos climáticos são de grande importância, devido a sua real dependência da agricultura,e o que exerce influência sobre sua capacidade de resiliência social mediante a tais crises (FAVERO E SARRIERA, 2014). E embora muitos programas do Governo tenham sido criados emergencialmente, a exemplo do Bolsa estiagem, que prevê atender os agricultores cujas plantações foram afetadas pela seca, Programa Operação CarroPipa, que teve como foco a distribuição de água potável à população rural ou construção de empreendimentos hídricos, que contribuem para o desenvolvimento socioeconômico local, como é o caso do Programa Um Milhão de Cisternas Rurais (P1MC), tais medidas devem ser compreendidas como forma paliativa e não emancipadora, visto que são emergenciais, do processo de vulnerabilidade social (COSTA et al., 2020).

Resiliência esta que depende da abundância de recursos do ecossistema, bem como a administração dos recursos em benefício de um coletivo (ADGER, 2000), o que justifica a percepção dos agricultores quanto a diversificação de atividades ocupacionais.

\section{CONCLUSÕES}

As percepções de risco e benefícios dos entrevistados constituem uma ferramenta integração dos conhecimentos tradicionais e fortalecimento de ações gestoras, visando a formulação de planos de manejo adequado para os sistemas naturais e sociais afetados pelo Projeto de Integração de Bacia Rio São Francisco com as Bacias Hidrográficas do Nordeste Setentrional (PISF). Uma vez que, possibilita identificar fatores de vulnerabilidade socioeconômico associados as R. gest. sust. ambient., Florianópolis, v. 10, n. 2, p. 189-208, jun. 2021. 
necessidades de adequações na gestão dos recursos hídricos. Podendo ainda destacar a necessidade de integração de diferentes atores sociais nas ações de tomada de decisão, uma vez que, podemos observar posicionamentos distintos em que, as mulheres apresentam uma preocupação mais holística do contexto socioambiental em comparação aos homens que se concentram mais nos fatores socioeconômicos. Enquanto, os benefícios apontados refletem a situação de vulnerabilidade dos entrevistados considerando o cenário de incertezas causados pelo histórico de secas na região.

\section{Referências}

AESA - Agência Executiva de Gestão das Águas do Estado da Paraíba, 2021. Disponível em: <https://www.aesa.pb.gov.br>. Accessed abril 2021.

ADGER, W. N. Social and ecological resilience: Are they related? Progress in Human Geography, v. 24, n. 3, p. 347-364, 2000.

AFROZ, R., BANNA, H., MASUD, M.M., AKHTAR, R., YAHAYA, S.R. Household's perception of water pollution and its economic impact on human health in Malaysia. Desalin. Water Treat., v. 57, p. 115-123; 2015.

ANA - Agência Nacional das Águas. Conjuntura dos recursos hídricos no Brasil 2017: relatório pleno. Brasília-DF: ANA, 2017. Disponível em: http://www.snirh.gov.br/portal/snirh/ centrais-de-conteudos/conjuntura-dos-recursoshidricos/conj2017_rel-1.pdf Acesso em: abril 2021.

ALBUQUERQUE, U. P.; CRUZ DA CUNHA, L. V. F.; LUCENA, R. F. P. Methods and techiques in ethnobiology and ethnoecology. New York: Springer Science, 2014. $480 \mathrm{p}$

ALEIXO, B.; REZENDE, S.; PENA, J. L.; ZAPATA, G.; HELLER, L. Direito humano em perspectiva : desigualdades no acesso à água em uma comunidade rural do nordeste brasileiro. Ambiente \& Sociedade, v. 19, n. 1, p. 63-82, 2015.

ALVARES, C. A., STAPE, J. L., SENTELHAS, P. C., DE MORAES GONÇALVES, J. L., \& SPAROVEK, G. Köppen's climate classification map for Brazil. Meteorologische Zeitschrift, v. 22, n. 6, p. 711-728, 2013.

ARTELL, J., AHTIAINEN, H., POUTA, E. Subjective vs. objective measures in the valuation of water quality. Journal Environmental Management, v.130, p. 288-296; 2013.

ASHLEY, C., HUSSEIN, K. Developing methodologies for livelihood impact assessment: experience of the African Wildlife Foundation in East Africa. Overseas Development London: Sustainable Livelihoods Working Paper, 2000.

BARBOSA, J. E. L.; SEVERIANO, J. S.; CAVALCANTE, H.; SILVA, D. L.; MENDES, C. F.; BARBOSA, V. V.; SILVA, R. D. S.; OLIVEIRA, D. A.; MOLOZZI, J. Impacts of

R. gest. sust. ambient., Florianópolis, v. 10, n. 2, p. 189-208, jun. 2021. 
inter-basin water transfer on the water quality of receiving reservoirs in a tropical semiarid region. Hydrobiologia, v. 848, p. 651-673, 2021.

BRASIL. Projeto de Integração do Rio São Francisco com Bacias Hidrográficas do Nordeste Setentrional: Relatório de Impacto Ambiental - RIMA. Brasil: Ministério da Integração Nacional, 2004. Disponível em: http://www.mi.gov.br/ documents/10157/3675235/ RIMA+JULHO+2004.pdf/78989068-cf76-4ab5-bf013b45473db7f9 Acesso em: abril 2021.

BURGER, J. et al. Attitudes and Perceptions about Ecological Resources and Hazards of People Living Aroung the Savannah River Site. Environmental Monitoring and Assessment, v. 57, n. January, p. 195-211, 1999.

BURNEY, J.; CESANO, D.; RUSSELL, J.; LA ROVERE, E. L.; CORRAL, T.; COELHO, N. S.; SANTOS, L. Climate change adaptation strategies for smallholder farmers in the Brazilian Sertão. Climatic Change, v. 126, p. 45-59, 2014.

CARVALHO, L. A.; MENANDRO, M. C. S. Representação da transposição do rio São Francisco na imprensa paraibana. Arquivos Brasileiros de Psicologia, v. 70, n. 1, p. 1-18, 2018.

CASTRO, C. N. Transposição do rio São Francisco: análise de oportunidade do projeto. Texto para Discussão. 2011. Disponível em: https://www.ipea.gov.br/portal/index.php?option=com_content\&view=article\&id=974.

Acesso em: abr. 2021.

CLARKE, K.R. e GORLEY, R.N. 2006 PRIMER v6: User manual/tutorial. Plymouth: PRIMER-E. 189p.

CONSÓRCIO ECOPLAN-SKILL. Projeto de Integração do Rio São Francisco com Bacias Hidrográficas do Nordeste Setentrional - Eixo Leste; Projeto Executivo do Lote D. Relatório Final do Projeto Executivo das Obras Complementares. Porto Alegre. 164 p. 2011.

COSTA, P. V. M.; OJIA, R. Transposição do rio São Francisco e a vulnerabilidade sociodemográfica: desafios ao bem-estar da população sertaneja. Revista Desenvolvimento e Meio Ambiente, v. 55, p. 141-165, 2020.

CRAUSBAY, S. D. RAMIREZ, A. R. Defining Ecological Drought for the Twenty-First Century. American Meteorological Society, v. 98, n. 12, p. 2543-2550, 2017.

DANTAS, J. C.; DA SILVA, R. M.; SANTOS, C. A. G. Drought impacts, social organization, and public policies in northeastern Brazil: a case study of the upper Paraíba River basin. Environmental Monitoring and Assessment, v. 192, n. 5, 2020.

ESPINOSA-GARCÍA, A.C., DÍAZ-ÁVALOS, C., GONZÁLEZ-VILLARREAL, F.J., VALSEGURA, R., MALVAEZ- OROZCO, V., MAZARI-HIRIART, M. Drinking water quality in a Mexico City University community: perception and preferences. EcoHealth, v.12, p. 88-97, 2015.

FAVERO, E.; SARRIERA, J. C. Impactos da seca no bem-estar psicológicode agricultores familiares do sul do Brasil. Temas em Psicologia, v. 22, n. 4, p. 809-822, 2014.

R. gest. sust. ambient., Florianópolis, v. 10, n. 2, p. 189-208, jun. 2021. 
GALLO, N. C.; NAVARRO, A. C. L. Mulher e Água: Definições e novas representações.Labor \& Engenho, v.12, n.2, p.166-181, 2018.

GUEDES, G. R. et al. [Risk of contamination from exposure to Rio Doce water: a case study on the population's perceptions in Tumiritinga, Minas Gerais State, Brazil]. Cadernos de saúde pública, v. 31, n. 6, p. 1257-68, 2015.

HANNIGAN, John. Sociologia Ambiental. Editora Vozes, 2009.

HENKE, S. L. Os Riscos Das Decisões: Um Estudo De Caso Da Transposição Do Rio São Francisco. Novos Estudos Jurí-dicos, v. 18, n. 2, p. 269, 2013.

JONES, R. E.; DUNLAP, R. E. The social bases of environmen- tal concern: Have they changed over time. Rural Sociology, v. 57, n. 1, p. 28-47, 1992.

MACHADO, L. W.; LA ROVERE, E. L. The Traditional Technological Approach and Social Technologies in the Brazilian Semiarid Region. Sustainability, v. 10, 2018.

MARENGO, J. A.; CUNHA, A. P.; ALVES, L. M. A seca de 2012-15 no semiárido do Nordeste do Brasil no contexto histórico. Climanálise, v. 3, p. 49-54, 2016

MELO, L. A. Relações de gênero na convivência com o semiárido brasileiro: a água para o consumo doméstico. In: SCOTT, P.; CORDEIRO, R. (Orgs). Agricultura familiar e gênero: Práticas, movimentos e políticas públicas. Recife: Ed. Universitária da UFRE, 2006.

MENDONÇA, F.; OLIVEIRA, I. M. D. Climatologia: noções básicas e climas do Brasil (p. 2007). São Paulo: Oficina de Texto, 2007.

MOURA, M.S.B.; GALVÍNCIO, J. D., BRITO, L.T.L., SOUZA, L.S. B., SÁ, I. I. S., SILVA, T. G. F. Clima e água de chuva no Semiárido. In: Brito, L. T. L., Moura, M. S. B., Gama, G. F. B., (Org.). Potencialidades da água de chuva no Semiárido brasileiro. Petrolina: Embrapa Semiárido, p.37-59, 2007.

NARAYAN, D., CHAMBERS, R., SHAH, M.K., PETESCH, P. Voices of the Poor: Crying Out for Change, Oxford University Press and World Bank, Oxford, 2000.

PEREIRA-JÚNIOR, J. S., 2007. Nova delimitacao do semi-arido brasileiro. Biblioteca Digital da Camara dos Deputados, Brasília.

QUINN, C. H. et al. Local perceptions of risk to livelihood in semi-arid Tanzania. Journal of Environmental Management, v. 68, n. 2, p. 111-119, 2003.

RAMBONILAZA, T.; JOALLAND, O.; BRAHIC, E. Landowner's perception of flood risk and preventive actions in estuarine environment: An empirical investigation, Journal of Environmental Management, v. 180, p. 272-279, 2016.

REBOUÇAS, A. C. Água na região Nordeste: Desperdício e escassez. Estudos Avançados, v.11, n. 29, p. 127-154, 1997.

SANTANA, F. A. Comunidades Ribeirinhas da Amazônia: Um relato de experiência. Perspectiva Amazômica, v. 6, n 3, p. 47-56, 2013.

Santos, R. V.; Vital, A. F. M. (Org.). Temas de impactos socioambientais em área do rio Paraíba. Maceió: TexGraf, 2017.

R. gest. sust. ambient., Florianópolis, v. 10, n. 2, p. 189-208, jun. 2021. 
SILVA, R. C. B. A. Participação social como estratégia de desenvolvimento local sustentável: estudo do reassentamento da Vila Produtiva Rural VPR Negreiros, no Projeto da Transposição do Rio São Francisco. 2015. Dissertação (Mestrado em Gestão Ambiental) - Programa de Pós-Graduação em Gestão Ambiental. Instituto Federal do Pernambuco, Recife, 2015.

SILVA, R. M.; SANTOS, C. A. G.; MARANHÃO, K. U. A.; SILVA, A. M. E.; LIMA, V. R. $P$. Geospatial assessment of eco- environmental changes in desertification area ofthe Brazilian semi-arid region. Earth Sciences Research Journal,v. 22,175- 186, 2018.

SILVA, M. M. V.; DINIZ, P. C. O.; MEDEIROS, P. C. Conflitos pelo acesso à água: impactos da Transposição do rio São Francisco sobre a Vila Lafayete, Monteiro/PB. Revista Desenvolvimento e Meio Ambiente, v. 55, p. 166-185, 2020.

SHI, X.; HE, F. The environmental pollution perception of residents in coal mining areas: A Case study in the hancheng mine area, Shaanxi Province, China. Environmental Management, v. 50, n. 4, p. 505-513, 2012.

TALLMAN, H.; BENEDICTO, M. Velho Chico Pede Passagem. Retratos. Revista do IBGE, v. 13, p. 6-11, 2018.

ZHOURI, A.; LASCHEFSKI, K. Conflitos ambientais. Grupo de Estudos em Temáticas Ambientais - GESTA/UFMG, 2014. Disponível em: $<$ https://conflitosambientaismg.lcc.ufmg.br/producao-academica/categoria/textosanaliticos-do-mapa--de-conflitos-ambientais/page/2/>. Acesso em: abril 2021.

R. gest. sust. ambient., Florianópolis, v. 10, n. 2, p. 189-208, jun. 2021. 